\title{
Real-Time ECG Monitoring for Patients with Implantable Pacemaker - A Review of Current Status
}

\author{
John Wang \\ Philips Healthcare, Andover, MA, USA
}

\begin{abstract}
Implantable pacemakers are widely used for managing patients with certain cardiac rhythm abnormalities to reduce mortality and morbidity while improving quality of life. However, for ECG devices that have to analyze the surface ECG signals, it has always been a major challenge to maintain a high level of performance. Several recent advances in pacemaker development have created even further challenges for the ECG devices.

Earlier developments such as ventricular pacing, atrial pacing, dual-chamber pacing, unipolar vs. bipolar pacing are well understood. However, more recent advances such as: biventricular pacing, multipoint pacing, leadless pacemakers, device-specific algorithms for reducing frequency of right ventricle pacing and managing pacing output to improve battery life are much more complex and thus are more difficult to evaluate whether the ECG devices can still function effectively.

To ensure continuous safe and effective monitoring, the basic requirements are: a) mandatory manufacture disclosure of device information required for monitoring algorithm design, b) development of up-to-date paced algorithm performance evaluation procedures, and c) a suitable paced database for algorithm development and testing. This would require the cooperation of pacemaker and ECG device manufactures, and regulatory agencies.
\end{abstract}

\section{Introduction}

Electrocardiographic monitoring, which allows for continuous non-invasive detection and documentation of cardiac arrhythmia, is one of the most frequently used monitoring procedures for managing in-hospital patients. Current commercial systems are designed to detect most of the ventricular arrhythmias and some of the atrial arrhythmias for patients of all age groups including patients with implantable pacemaker [1].

Effective monitoring of paced patients has always been a challenge for ECG monitoring devices. In addition to the detection of all non-paced arrhythmias, several pacing related failures also need to be detected, including: loss of capture, failure-to-pace (oversensing), and failure- to-sense (undersensing). To provide effective paced monitoring, ECG devices must be able to detect pace pulses and intrinsic QRS complexes with high degree of sensitivity and specificity. To accomplish this, a complete understanding of the pace pulse characteristics and their interaction with patients' intrinsic rhythms is required.

Recent advances in pacing technologies [2] such as biventricular pacing, multipoint/multisite pacing, leadless pacemakers and many device-specific algorithms for reducing frequency of right ventricle pacing and managing pacing output to improve battery life are much more complex and thus are more difficult to assess the effectiveness of the ECG devices for paced patient monitoring. Existing specifications of the pace pulses and testing requirements [3] have not been updated to cover these new advances in pacing technologies.

The objectives of this paper is to provide an overview of the paced patient monitoring objectives, review of advances in pacing device development that have impact on the continuing development of effective paced patient monitoring algorithms. Finally, several key issues are described and recommendations are provided on how to address them moving forward to ensure that effective paced patient monitoring can be achieved.

\section{Paced patient monitoring}

\subsection{Monitoring objectives}

In addition to the detection of all the non-pacemaker related cardiac rhythm disturbances and changes, several additional paced-specific beats also need to be detected and classified as illustrated in Fig. 1:

Paced beat - There are three types of paced beats: atrial (Fig. 1a), ventricular (Fig. 1b), and AV sequential (dualchamber) paced (Fig. 1c).

Fusion beat - Fusion beats happen when an intrinsically conducted beat and a pace triggered beat occur simultaneously. Depending on the relative timing between the intrinsic beat and the paced beat, the QRS morphology can vary widely (Fig. 1d, beat \#1).

Pseudofusion beat - An ineffective pace pulse occurs near or in an intrinsic beat. Because the pace stimulus occurs 
after the heart has already depolarized, the pacemaker pulse is ineffective (Fig. 1d, beat \#4). Usually there is no major distortion of the QRS morphology unless the intrinsic QRS is very narrow.

Pseudopseudofusion beat - A superimposition of an atrial stimulus on an intrinsic QRS complex. The pacing is ineffective since the atrial pacing pulse cannot contribute to the ventricular contraction.

Anti-tachycardia pacing - Fast multiple pacing pulses higher than the patient's intrinsic rate are delivered to interrupt a tachyarrhythmia episode and restore the patient's normal rhythm (Fig. 1e).

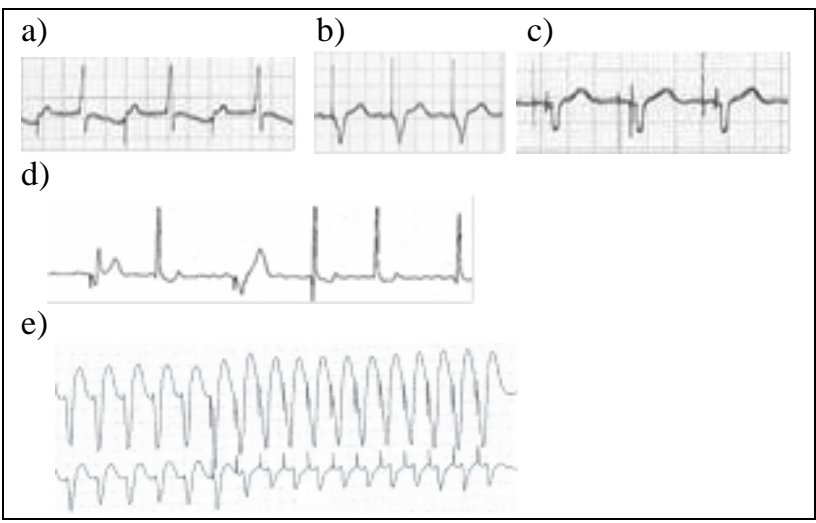

Figure 1. ECG examples of (a) atrial pacing, (b) ventricular pacing, (c) AV sequential pacing, (d) paced fusion (beat 1), intrinsic beats (beats 2, 5, 6), paced beat (beat 3), pseudofusion beat (beat 4), and (e) antitachycardia pacing (2-channel recording).

The important pacing-specific events of interest in monitoring paced patients are shown in Fig. 2:

Oversensing - Oversensing, also called failure to pace, occurs when the pulse generator is inappropriately inhibited due to the detection of unwanted electrical signals (such as myopotentials, EMI, T-waves or crosstalk between atrial and ventricular channels in dualchamber pacemakers). The oversensed signal may not be visible on the surface ECG (Fig. 2a).

Undersensing - It occurs when the pacemaker fails to sense the intrinsic $\mathrm{R}$ waves and inappropriately timed, asynchronous output pulses are delivered. Fig. $2 \mathrm{~b}$ shows a paced beat (beat \#3) due to undersensing, and Fig. 2c shows a PVC with a pacing pulse in the T-wave region.

Failure to capture - It occurs when the pacing stimulus fails to initiate myocardial depolarization. Some typical causes are threshold rise, lead displacement, or lead dislodgement. On the surface ECGs, pacing spikes are present, but not followed by a captured QRS complex. Fig. $2 d$ shows pacing non-capture with low heart rate and Fig. 2e shows that the patient is in asystole.

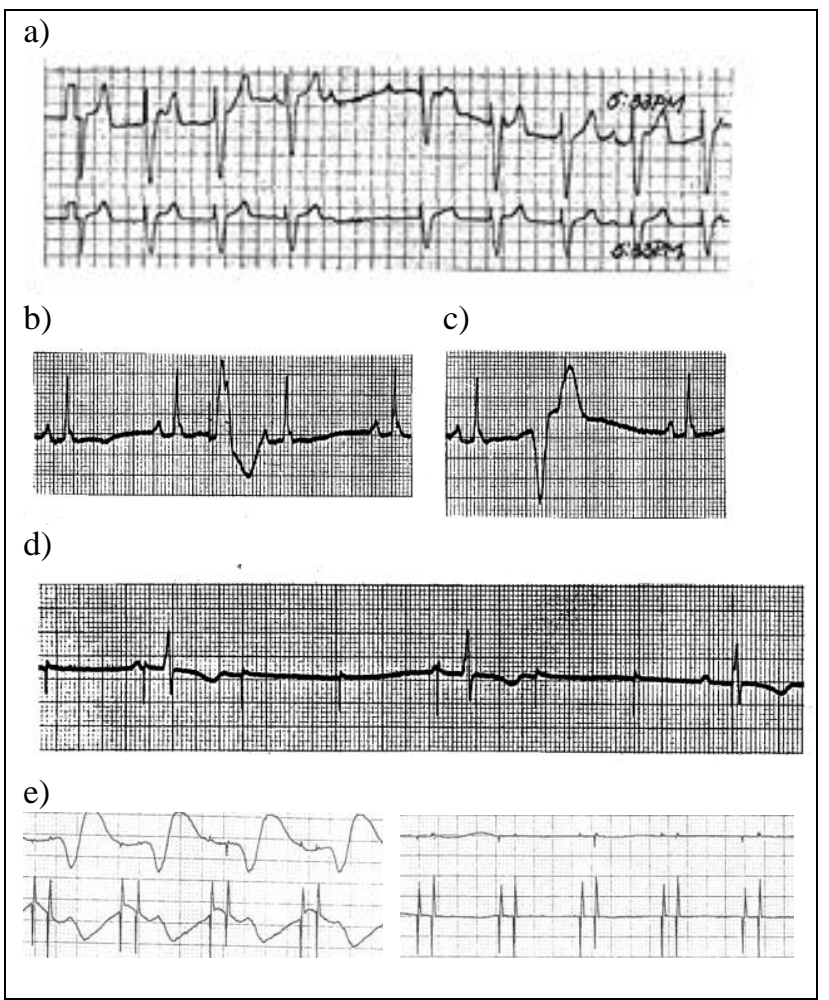

Figure 2. Examples of pacing-specific events: a) pacer not pacing (oversensing), b) and c) Pacer not sensing (undersensing), d) pacer not capture with intrinsic rhythm, e) 2-channel recording of pacer not capture without intrinsic rhythm (asystole).

\subsection{Pace pulse characteristics}

A pace pulse waveform from a constant-voltage capacitor-discharged output pacemaker consists of a main pulse and followed by a repolarization pulse (also called overshoot) with opposite polarity as shown in Fig. 3. The main pulse is used to stimulate the heart and is characterized by its narrow width (W), sharp rise (slew rate, $\mathrm{dV} / \mathrm{dT}$ ), and large variation in amplitude (A). The drop on the voltage of the main pulse depends on the capacitor size of the output circuit. The repolarization pulse is characterized by its amplitude (U), and recharge

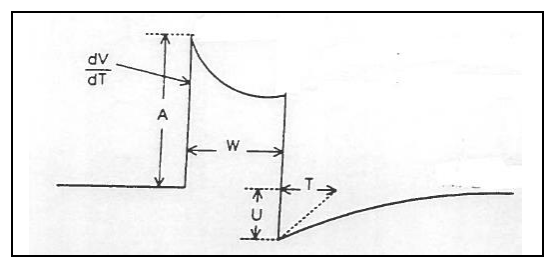

Figure 3. A typical pace pulse consists of a main pulse and a repolarization pulse with opposite polarity. 
time constant $(\mathrm{T})$ and is used to deplete the charge built up around the tip of the lead to allow the lead to sense the cardiac activity. The values of these parameters can vary widely [3] depending on many factors including the pacemaker pacing configuration as described below.

\subsection{Pacing configuration: unipolar vs. bipolar}

For unipolar pacing, the impulse flows through the electrode tip (cathode) to stimulate the heart and return through the body tissue to the generator (anode). It produces large pacing pulses that are difficult to analyze (Fig. 4). The non-captured pace pulses (waveform \#1 and \#3) are difficult to differentiate from the three captured beats at the end. In bipolar pacing, the lead also has a ring to serve as the anode. The resultant pace pulses are small and in some cases very difficult to see (Fig. 5). Most modern pacemakers offer programmable pacing configuration and are able to automatically reconfigure from bipolar to unipolar pacing if one of the bipolar electrode becomes too poor to provide effective pacing.

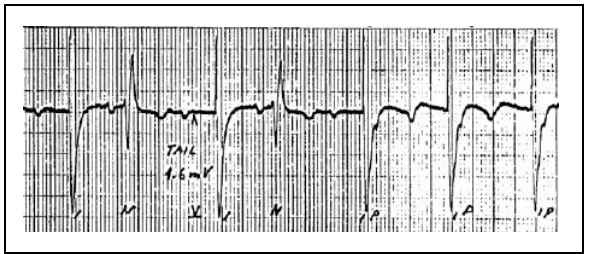

Figure 4. An example of large unipolar pace pulses.

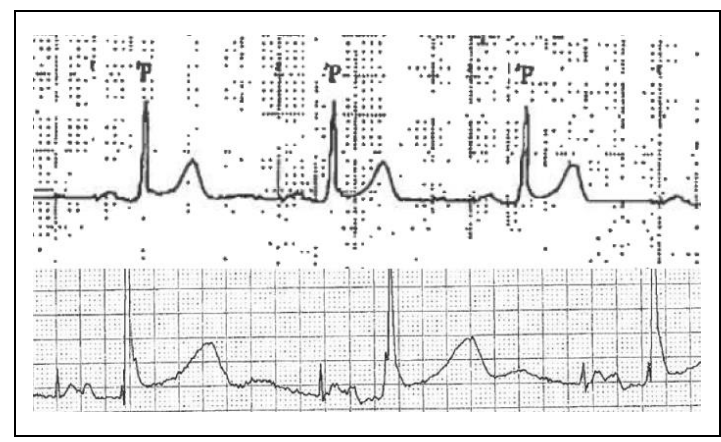

Figure 5. An example of paced pseudofusion beats with small bipolar pace pulses: top channel low sampling rate, lower channel high sampling rate.

To overcome the problem of not being able to see the small bipolar pulses, many ECG devices insert artificial vertical lines with fixed amplitude to the ECG signal at the points where pace pulses are detected. The problem with this approach is that the resultant ECG will not be usable if there are too many false triggers from the pace pulse detector (Fig. 6). To reduce the incidence of this problem, most ECG devices require users to set whether the patient is paced or not such that this problem can only occur on the smaller subset of paced patients only.

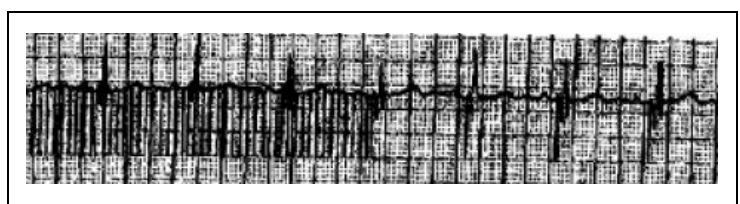

Figure 6. An example of artificially inserted pacing spikes on the ECG signals caused by false positive triggers of the paced pulse detector of the ECG device.

\subsection{Advanced pacing development}

1) Biventricular pacing - To improve treatment of heart failure patients, pacemakers with the capability of supporting a left ventricle pacing lead placement have been developed. The delay between RV and LV pacing can be adjusted to optimize performance for each patient. Both ventricular pace pulses remain unchanged when the programed delay is large. However, when the delay becomes smaller, the repolarization pulse associated with the first ventricular pacing pulse will start to change. For one device (Fig. 7), when the delay is set to $20 \mathrm{~ms}$ or less, it will hold off the repolarization pulse associated with the $1^{\text {st }}$ pacing pulse until $20 \mathrm{~ms}$ after the $2^{\text {nd }}$ ventricular pace pulse. Since the exact behavior is likely to be device dependent, designing a monitoring algorithm that works well with all possible settings could be very challenging.

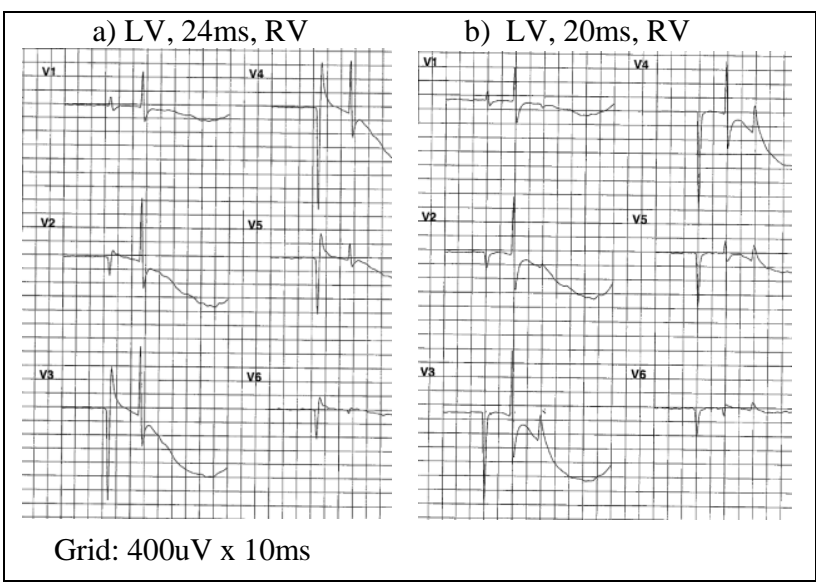

Figure 7. Leads V1-V6 from a biventricular pacing device with delayed repolarization pulses.

2) Multisite/multipoint pacing - Multisite pacing has been developed to overcome the non-response problem from many patients with the conventional biventricular 
pacing. Multisite pacing is achieved by the use of multiple leads placed within the coronary sinus (dual-vein pacing) or the use of multipolar left ventricular pacing leads which can deliver pacing stimuli at multiple sites within the same vein. Both methods allow the pacing of two left ventricle sites in addition to the right ventricle. The delays between these multiple pacing pulses can also be adjusted to achieve optimal performance. How to best optimize these newly expanded pacing configurations remains to be determined through on-going clinical trials. In addition, how these more complex pacing configurations impact the characteristics of the pace pulses on the surface ECG also need to be thoroughly studied.

3) Leadless pacemaker - A major shift from the traditional pacemaker system is the development of the leadless pacemakers [2] where the self-contained pulse generator has built-in battery and electrode for implantation in the right ventricular chamber via a transfemoral catheter approach. Advantages of a leadless pacemaker include the avoidance of a surgical scar and pacemaker pocket, risk of infections, and problems with lead placement.

The potential problems for monitoring devices are: 1) the pace pulses on the surface ECG may be even smaller than those from the traditional pacemakers. Thus, to detect them the sensitivity of the pace pulse detector of the ECG device must be increased. As such, there will be more false positive triggers due to noise interferences, 2) with the pacemaker placed inside the heart chamber clinicians may not be able to verify easily whether the monitored patient has an implantable pacemaker or not in order to set correctly the monitoring mode (Paced or Nonpaced) for effective monitoring.

\section{Issues and recommendations}

1) Pacemaker manufactures disclosure -.Due to the large number of available pacemakers on the market from different manufactures with numerous device-specific features that can be programed/adjusted over a wide range of parameter settings, it is becoming impossible for the ECG device companies to keep track and gather the relevant device information that are critical in the design of their paced patient monitoring algorithms. Recommendation to address this issue is to require pacemaker manufactures to disclose the relevant information with sample surface ECG waveforms. This disclosure should be publically available and easily assessable. To ensure this is done, the recommendation is to make this a mandatory requirement for regulatory device clearance.

2) ECG testing standard development - Current standards for paced algorithm testing as described in [3] was developed in the earlier 80s. Updating the document is desirable but difficult due to the lack of sufficient information on the current pacemakers. If the recommendation of manufacture disclosure as described is followed, it is expected that over a period of time there will be enough information gathered for the AAMI ECG standard working group to develop a plan for a major update.

3) Paced database development - Unlike the situation for non-paced patients where many public data bases are readily available, there are no publically available data bases for paced patients. Although several data bases do contain paced patients, the paced ECGs do not preserve pace pulses during digitization and therefore are not usable. Therefore, a high-fidelity dataset contains examples of paced ECG waveforms from a variety of pacemakers in different pacing configurations and parameter settings is needed for further development and testing of paced ECG monitoring algorithms. Due the large scope of this effort, it is recommended that all ECG device manufactures should work together to develop such a publically available dataset.

\section{Conclusion}

Technology advances in pacemaker development have made effective monitoring of pacemaker patients a major challenge due to the lack of a) pacemaker information required for algorithm design, $b$ ) paced testing procedures for performance validation, and c) paced database for algorithm development and validation. Recommendations to address these identified issues would require the cooperation of all pacemaker and ECG device manufactures, clinical users, and regulatory agencies.

\section{References}

[1] Epstein EA, DiMarco JP, Ellenbogen KA, et al. Practice Guideline: 2012 ACCF/AHA/HRS focused update incorporated into the ACCF/AHA/HRS 2008 guidelines for device-based therapy of cardiac rhythm abnormalities. JACC 2013;61:e6-75. [2] Mulpuru SK, Madhavan M, Mcleod CJ, et al. Part I Cardiac pacemakers: Function, troubleshooting, and management. Part II - Advances and future directions in cardiac pacemakers. JACC 2017;69:189-235.

[3] ANSI/AAMI/IEC 60601-2-27 Medical electrical equipment - Part 2-27: Particular requirements for the basic safety and essential performance of electrocardiographic monitoring equipment.

Address for correspondence:

John Wang

Philips Healthcare, MS-4308

3000 Minuteman Road

Andover, MA 01810-1099, USA

E-mail: john.j.wang@philips.com 